\title{
Polarimetric Distance-Dependent Models For Large Hall Scenarios
}

\author{
Shiqi Cheng, Davy P. Gaillot, Member, IEEE, Emmeric Tanghe, Member, IEEE, Pierre Laly, Thierry Demol, \\ Wout Joseph, Senior Member, IEEE, Luc Martens, Member, IEEE, and Martine Liénard
}

\begin{abstract}
A comprehensive polarimetric distance-dependent model of the power delay profile (PDP) and path gain is proposed. The model includes both Specular Multipath Components (SMC) and Dense Multipath Components (DMC); the latter being modeled with an exponential and power law. The parameters of the model were estimated from polarimetric measurements of a large hall radio channel under Line-Of-Sight (LOS) conditions at $1.3 \mathrm{GHz}$ with a dedicated procedure. The validity and robustness of the proposed approach is provided by the good agreement between the polarimetric data and models for the investigated transmitter-receiver distance range. Furthermore, the description of the radio channel with path loss models is discussed for cases where the DMC is included and a two-step method to compute the path loss characteristics directly from the measured data is developed. The results of this contribution highlight the fact that a complete polarimetric description of all propagation mechanisms and related path loss models is desired to design faithful polarimetric radio channel models.
\end{abstract}

Index Terms-Polarimetric measurement, Distance-dependent channel model, Indoor environment, Dense multipath component, path loss model.

\section{INTRODUCTION}

$\mathbf{O}$ VER the recent years, extensive experimental studies have been performed with the aim of providing a deeper physical comprehension of the propagation mechanisms and developing physically-sound radio channel models for all types of scenarios [1]. Originally, the radio channel was commonly considered as a mere collection of Specular Multipath Components (SMC) that have well-defined discrete locations in the different radio channel dimensions (e.g., space, frequency, time, etc.). Furthermore, distributed diffuse scattering on electrically small objects and SMC with low signal-to-noise ratio (SNR) values are also included into the channel but were historically interpreted as polluting noise. Nowadays, it is understood that these effects cannot be distinguished from each other and form the basis for the Dense Multipath Components (DMC). In other words, the DMC can alternatively be interpreted as the non-coherent superposition of paths with weaker SNRs than the SMC, which still follow the specular power decay as a function of distance [2], [3]. Hence, the introduction

S. Cheng, D. P. Gaillot, P. Laly, and M. Liénard are with the University of Lille I, IEMN, Batiment P3, Villeneuve d'Ascq, 59655 FR, e-mail: davy.gaillot@univlille1.fr.,

T. Demol is with CITC-EuraRFID, 165 Avenue de Bretagne, Lille, 59000 FR, e-mail: tdemol@ citc-eurarfid.com.

E. Tanghe, L. Martens, and W. Joseph are with Ghent University/iMinds, Department of Information Technology, B-9050 Ghent, Belgium, e-mail: emmeric.tanghe@intec.ugent.be.

Manuscript received January 23, 2015; revised xx xx, 2015. of DMC in the physical model implies that common radio channel parameters have to be re-evaluated. Indeed, faithful models of the DMC are critical to reproduce the propagation characteristics of the radio channel in indoor scenarios such as the path loss, mean delay, root mean squared (rms) delay spread. These characteristics are used for typical coverage analysis, network optimization, localization [4], or even human exposure analysis [5].

To this end, various DMC models were developed for indoor environments [6], [7], [8], [9] from the room electromagnetics theory for diffuse scattering observed in reverberation chambers [10], [11]. In those models, the DMC is typically observed as the decaying slope of the power delay profile (PDP) [12] and characterized by its reverberation time [8]. It was shown that the energetic contribution of the DMC to the radio channel can vary between $20 \%$ and $80 \%$ for indoor or industrial scenarios [2], [13], [14], [15]. A distance-dependent model was recently reported for indoor radio channels where the PDP is described as the sum of the early SMC part of the PDP and reverberant component [16]. The early part or primary SMC includes the Line-Of-Sight (LOS), if present, and first-order reflections off the walls, floor, ceiling, and objects. Similarly to the room electromagnetics theory, the reverberant component is the remaining part of the PDP such that the DMC cannot be physically distinguished from the secondary SMC (high-order reflections, diffractions, etc.).

Modern radio channel models are expected to encompass a polarimetric description of the physical radio link that could be used for the optimization of diversity-based wireless communications or dedicated applications. In our previous work, the general radio characteristics, XPD statistics, and propagation mechanisms were investigated from each estimated individual SMC and DMC as a function of distance and shadowing conditions [17]. Inspite being appealing for the propagation community, polarimetric PDP and path gain models as well as path loss models including both the SMC and DMC are missing in the literature. Here, the contribution of the paper aims at filling this gap and is two-fold:

1) First of all, a polarimetric model of the SMC and DMC $\mathrm{PDP} /$ path gain is developed. The proposed model is a strongly modified extension of [16]. In particular, it provides a finer polarimetric description of the SMC which are decomposed into primary and secondary parts and the DMC which is modeled with an exponential and power law. The parameters of the model are estimated from Multiple-Input Multiple-Output (MIMO) radio channel measurements in a large hall with dedicated processing steps and for larger distances compared 
to the original paper. The polarimetric path gain model can be used to describe any general characteristics such as the rms delay spread, XPD, or DMC fractional power. From this approach, single-slope path loss models are discussed to take into account the contribution of the DMC.

2) Furthermore, an additional important novelty of this work is the development of a two-step fitting method to obtain jointly the path loss characteristics for the primary SMC and DMC directly from the measured channels. This approach is validated with the measurement and modeled data. A connection between the DMC path loss characteristics and room electromagnetics theory is also highlighted.

The paper is organized as follows: the polarimetric distancedependent PDP and path gain models are proposed in Section II. Section III presents the measurement scenario and the data processing is carefully detailed. The parameters of the model and it validation are discussed in Section IV. Before concluding, SMC plus DMC path loss models are derived based on the observation of the reverberation properties. Finally, a two-step fitting method is proposed to obtain the path loss characteristics for the primary SMC and DMC from the measured data.

\section{Polarimetric PDP And Path Gain Modeling}

\section{A. PDP Model}

From the modeling point of view, the distance-dependent PDP can be understood as the sum of the band-limited SMC and DMC PDP:

$$
\mathbf{G}(\tau, d)=\mathbf{G}_{S M C}(\tau, d)+\mathbf{G}_{D M C}(\tau, d) .
$$

Steinbock et al. [16] recently proposed to decompose the PDP into a primary and reverberant component which both display a dependence to the distance:

$$
\mathbf{G}(\tau, d)=\mathbf{G}_{p r i}(\tau, d)+\mathbf{G}_{r e v}(\tau, d) .
$$

The primary component describes the early part of the PDP and includes the LOS and first-order reflections (if present) off the floor, ceiling, walls, and objects. It was proposed to be simply modeled by:

$$
\mathbf{G}_{p r i}(\tau, d)=\mathbf{G}_{0, p r i}\left(\frac{d_{0}}{d}\right)^{n_{p r i}} \delta\left(\tau-\frac{d}{c}\right),
$$

where $n_{\text {pri }}$ is the path loss exponent, $\mathbf{G}_{0, \text { pri }}$ the reference gain for the primary component at reference distance $d=d_{0}$, and $c$ the speed of light. In contrast, the reverberation component is the remainder of the channel after the primary component has been removed and describes the exponentially decaying behavior of the PDP tail as:

$$
\mathbf{G}_{r e v}(\tau, d)=\mathbf{G}_{0, r e v} e^{-\tau / \mathbf{T}}, \tau>\frac{d}{c}
$$

where $\mathbf{T}$ is the reverberation time [8], [10]. From this point of view, it can be seen that the reverberation component is the sum of the DMC [12] and the secondary SMC (high-order reflections, diffractions, etc.):

$$
\mathbf{G}_{r e v}(\tau, d)=\mathbf{G}_{s e c}(\tau, d)+\mathbf{G}_{D M C}(\tau, d) .
$$

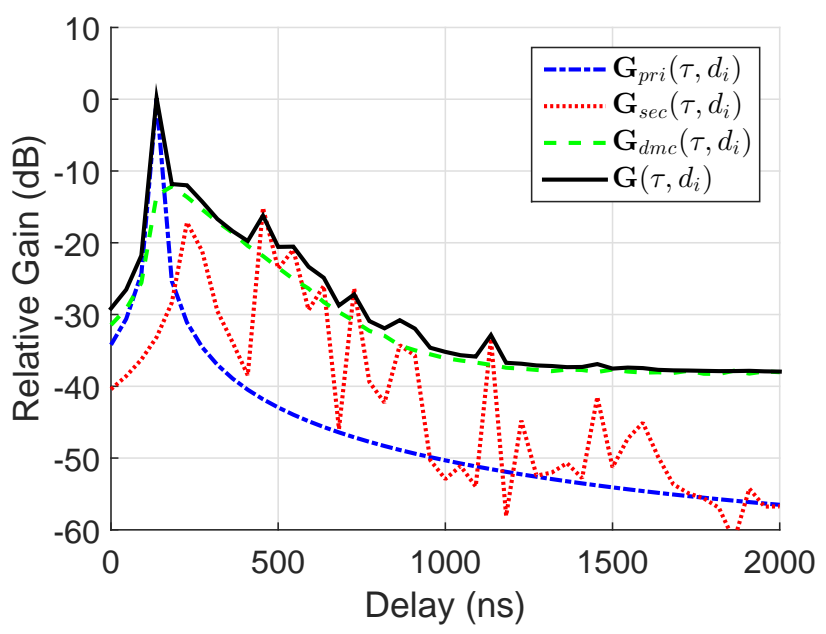

Fig. 1. Illustration of the PDP behavior in an indoor scenario for a given transmitter-receiver distance $d_{i}$. The PDP includes the primary SMC, secondary SMC, and DMC.

This modeling decomposition was shown to produce faithful distance-dependent radio characteristics (mean delay, delay spread, path gain, and kurtosis). However, the propagation properties and mechanisms of the DMC and secondary SMC could not be individually assessed. Here, we propose to alleviate this problem by introducing the following decomposition for the PDP:

$$
\mathbf{G}(\tau, d)=\mathbf{G}_{p r i}(\tau, d)+\mathbf{G}_{s e c}(\tau, d)+\mathbf{G}_{D M C}(\tau, d) .
$$

As an example, the decomposition of the PDP into the primary/secondary SMC, and DMC is illustrated in this figure in an indoor scenario for a given transmitter-receiver distance $d_{i}$. As illustrated in Fig. 1, the secondary SMC includes a wide collection of high-order SMCs that have different time-delays and gains. It can simply be modeled as the superposition of weighted single-Dirac terms with a power law model like with the primary component (see Eq. 3):

$$
\mathbf{G}_{s e c}(\tau, d)=\sum_{k=1}^{N_{s e c}} \mathbf{G}_{k, s e c}\left(\frac{d_{0}}{d}\right)^{n_{k, s e c}} \delta\left(\tau-\tau_{k}(d)\right),
$$

where $n_{k, \text { sec }}$ and $\tau_{k}(d)$ are the path loss exponent and distance-dependent time-delay for the $k^{t h}$ secondary SMC, respectively. $\mathbf{G}_{k, \text { sec }}$ is the reference gain at reference distance $d=d_{0}$ for the $k^{t h}$ secondary SMC and $N_{\text {sec }}$ is the number of SMC. In a simple empty environment such as the investigated one, it is assumed that $N_{\text {sec }}$ does not change with the distance.

The exponential function from Eq. (4) was kept to model the DMC in the delay domain:

$$
\mathbf{G}_{D M C}^{E x p}(\tau, d)=\mathbf{G}_{0, D M C}^{E x p} e^{-\tau / \mathbf{T}}, \tau>\frac{d}{c},
$$

where $\mathbf{G}_{0, D M C}^{P o w}$ is the reference gain at reference distance $d=$ $d_{0}$. For the sake of comparison, it is also suggested to use a power-law to model each non-coherent path contributing to the DMC. Indeed, the development of path loss models are always performed with this model and it can be useful to compare with already published path loss results for radio channels where 
DMC was identified as the strongest component. However, the authors would like to emphasize that the connection to room electromagnetics theory is completely missing when a power law is used.

$$
\mathbf{G}_{D M C}^{P o w}(\tau, d)=\sum_{k=1}^{N_{D M C}} \mathbf{G}_{k, D M C}\left(\frac{d_{0}}{d}\right)^{n_{k, D M C}} \delta\left(\tau-\tau_{k}(d)\right),
$$

where $n_{k, D M C}$ is the path loss exponent and $\mathbf{G}_{k, D M C}$ is the reference gain for the DMC at reference distance $d=d_{0}$ for the $k^{t h}$ path, respectively. $N_{D M C}$ is the number of paths for the DMC. Moreover, when polarimetric measurements are considered, all components can be further decomposed into a co-polar and cross-polar component :

$$
\mathbf{G}^{X Y}(\tau, d)=\mathbf{G}_{p r i}^{X Y}(\tau, d)+\mathbf{G}_{\text {sec }}^{X Y}(\tau, d)+\mathbf{G}_{D M C}^{X Y}(\tau, d),
$$

where the subscripts $X$ and $Y$ denote the polarization of the transmitting and receiving antenna, respectively. $X$ and $Y$ are either horizontal $(H)$ or vertical $(V)$. This decomposition is motivated by the fact that electromagnetic waves might suffer strong depolarization mechanisms in highly-reflective environments like indoor scenarios. Necessarily, all components will experience depolarization effects but at different scales due to the nature of each propagation mechanism. Hence, the proposed distance-dependent polarimetric model for the primary SMC is given by:

$$
\begin{gathered}
\mathbf{G}_{p r i}^{H H}(\tau, d)=\chi_{p r i}^{H H}(d) \mathbf{G}_{0, p r i}^{H H}\left(\frac{d_{0}}{d}\right)^{n_{p r i}^{H H}} \delta\left(\tau-\frac{d}{c}\right), \\
\mathbf{G}_{p r i}^{H V}(\tau, d)=\left(1-\chi_{p r i}^{H H}(d)\right) \mathbf{G}_{0, p r i}^{H H}\left(\frac{d_{0}}{d}\right)^{n_{p r i}^{H V}} \delta\left(\tau-\frac{d}{c}\right), \\
\mathbf{G}_{p r i}^{V V}(\tau, d)=\chi_{p r i}^{V V}(d) \mathbf{G}_{0, p r i}^{V V}\left(\frac{d_{0}}{d}\right)^{n_{p r i}^{V V}} \delta\left(\tau-\frac{d}{c}\right), \\
\mathbf{G}_{p r i}^{V H}(\tau, d)=\left(1-\chi_{p r i}^{V V}(d)\right) \mathbf{G}_{0, p r i}^{V V}\left(\frac{d_{0}}{d}\right)^{n_{p r i}^{V H}} \delta\left(\tau-\frac{d}{c}\right),
\end{gathered}
$$

where $\chi_{p r i}^{H H}$ and $\chi_{p r i}^{V V}$ are the distance-dependent polarization coefficients for $H H$ and $V V, \mathbf{G}_{0, p r i}^{H H}$ and $\mathbf{G}_{0, p r i}^{V V}$ the gain at reference distance $d=d_{0}$ for $H H$ and $V V$, and $n_{\text {pri }}^{X Y}$ the path loss exponent for each polarization link.

The distance-dependent polarimetric model for the secondary SMC is given by:

$$
\begin{aligned}
\mathbf{G}_{\text {sec }}^{H H}(\tau, d) & =\sum_{k=1}^{N_{s e c}} \chi_{k}^{H H}(d) \mathbf{G}_{k, s e c}^{H H}\left(\frac{d_{0}}{d}\right)^{n_{s e c, k}^{H H}} \delta\left(\tau-\tau_{k}(d)\right), \\
\mathbf{G}_{s e c}^{H V}(\tau, d) & =\sum_{k=1}^{N_{s e c}}\left(1-\chi_{k}^{H H}(d)\right) \mathbf{G}_{k, s e c}^{H H}\left(\frac{d_{0}}{d}\right)^{n_{s e c, k}^{H V}} \delta\left(\tau-\tau_{k}(d)\right), \\
\mathbf{G}_{s e c}^{V H}(\tau, d) & =\sum_{k=1}^{N_{s e c}}\left(1-\chi_{k}^{V V}(d)\right) \mathbf{G}_{k, s e c}^{V V}\left(\frac{d_{0}}{d}\right)^{n_{s e c, k}^{V H}} \delta\left(\tau-\tau_{k}(d)\right), \\
\mathbf{G}_{s e c}^{V H}(\tau, d) & =\sum_{k=1}^{N_{s e c}} \chi_{k}^{V V}(d) \mathbf{G}_{k, s e c}^{H H}\left(\frac{d_{0}}{d}\right)^{n_{s e c, k}^{V V}} \delta\left(\tau-\tau_{k}(d)\right),
\end{aligned}
$$

where $\chi_{k}^{H H}$ and $\chi_{k}^{V V}$ are the distance-dependent polarization coefficients for $H H$ and $V V$ whereas $\mathbf{G}_{k, s e c}^{H H}$ and $\mathbf{G}_{k, \text { sec }}^{V V}$ are the reference gain for $H H$ and $V V$ at reference distance $d=$ $d_{0}$ for the $k^{t h}$ SMC. Finally, $n_{k, \text { sec }}^{X Y}$ is the path loss exponent for the $k^{t h}$ SMC for each polarization link.

Similar equations can be derived for the DMC with power law model whereas the distance-dependent polarimetric model for the DMC with exponential model is given by:

$$
\begin{aligned}
& \mathbf{G}_{D M C}^{H H}(\tau, d)=\chi_{D M C}^{H H}(d) \mathbf{G}_{0, D M C}^{H H} e^{-\tau / \mathbf{T}^{H H}}, \tau>\frac{d}{c}, \\
& \mathbf{G}_{D M C}^{H V}(\tau, d)=\left(1-\chi_{D M C}^{H H}(d)\right) \mathbf{G}_{0, D M C}^{H H} e^{-\tau / \mathbf{T}^{H V}}, \tau>\frac{d}{c}, \\
& \mathbf{G}_{D M C}^{V V}(\tau, d)=\chi_{D M C}^{V V}(d) \mathbf{G}_{0, D M C}^{V V} e^{-\tau / \mathbf{T}^{V V}}, \tau>\frac{d}{c}, \\
& \mathbf{G}_{D M C}^{V H}(\tau, d)=\left(1-\chi_{D M C}^{V V}(d)\right) \mathbf{G}_{0, D M C}^{V V} e^{-\tau / \mathbf{T}^{V H}}, \tau>\frac{d}{c},
\end{aligned}
$$

where $\chi_{D M C}^{H H}$ and $\chi_{D M C}^{V V}$ are the distance-dependent polarization coefficients for $H H$ and $V V, \mathbf{G}_{0, D M C}^{H H}$ and $\mathbf{G}_{0, D M C}^{V V}$ the gain at reference distance $d_{0}$ for $H H$ and $V V$, and $\mathbf{T}^{X Y}$ is the reverberation time for each polarization link. For the case where the room electromagnetics theory is valid, $\mathbf{T}^{X Y}$ is linked to the geometrical configuration of the scenario and should not depend on the distance. Whereas Eq. (6) can provide a general description of the radio channel characteristics such as the path loss, mean delay, or rms delay spread for example, the set of equations (11) to (22) provide a deeper understanding of the propagation mechanisms of the polarimetric SMC and DMC.

\section{B. Path Gain Model}

From the developed distance-dependent polarimetric PDP model, the path gain $\mathbf{P}^{X Y}$ averaged at distance $d$ is derived by integrating the PDP with respect to delay for each propagation mechanism and polarization state:

$$
\mathbf{P}^{X Y}(d)=\int \mathbf{G}^{X Y}(\tau, d) d \tau .
$$

Consequently, the following path gain models are obtained for $H H, H V, V H$, and $V V$, respectively:

$$
\begin{aligned}
& \mathbf{P}^{H H}(d)=\underbrace{\chi_{p r i}^{H H}(d) \mathbf{G}_{0, p r i}^{H H}\left(\frac{d_{0}}{d}\right)^{n_{p r i}^{H H}}}_{\mathbf{P}_{p r i}^{H H}(d)}+ \\
& \underbrace{\chi_{s e c}^{H H}(d) \mathbf{G}_{0, s e c}^{H H}\left(\frac{d_{0}}{d}\right)^{n_{s e c}^{H H}}}_{\mathbf{P}_{s e c}^{H H}(d)}+ \\
& \underbrace{\chi_{D M C}^{H H}(d) \mathbf{G}_{0, D M C}^{H H} \mathbf{T}^{H H} e^{\frac{-d}{c \mathbf{T}^{H H}}}}_{\mathbf{P}_{D M C}^{H H}(d)}, \\
& \mathbf{P}^{H V}(d)=\underbrace{\left(1-\chi_{p r i}^{H H}(d)\right) \mathbf{G}_{0, p r i}^{H H}\left(\frac{d_{0}}{d}\right)^{n_{p r i}^{H V}}}_{\mathbf{P}_{p r i}^{H V}(d)}+ \\
& \underbrace{\left(1-\chi_{s e c}^{H H}(d)\right) \mathbf{G}_{0, s e c}^{H H}\left(\frac{d_{0}}{d}\right)^{n_{s e c}^{H V}}}_{\mathbf{P}_{s e c}^{H V}(d)}+ \\
& \underbrace{\left(1-\chi_{D M C}^{H H}(d)\right) \mathbf{G}_{0, D M C}^{H H} \mathbf{T}^{H V} e^{\frac{-d}{c \mathbf{T}^{H V}}}}_{\mathbf{P}_{D M C}^{H V}(d)},
\end{aligned}
$$




$$
\begin{aligned}
& \mathbf{P}^{V H}(d)=\underbrace{\left(1-\chi_{p r i}^{V V}(d)\right) \mathbf{G}_{0, p r i}^{V V}\left(\frac{d_{0}}{d}\right)^{n_{p r i}^{V H}}}_{\mathbf{P}_{p r i}^{V H}(d)}+ \\
& \underbrace{\left(1-\chi_{\text {sec }}^{V V}(d)\right) \mathbf{G}_{0, \text { sec }}^{V V}\left(\frac{d_{0}}{d}\right)^{n_{\text {sec }}^{V H}}}_{\mathbf{P}_{\text {sec }}^{V H}(d)}+
\end{aligned}
$$

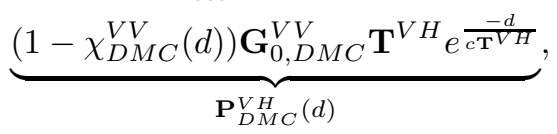

$$
\begin{aligned}
& \mathbf{P}^{V V}(d)=\underbrace{\chi_{p r i}^{V V}(d) \mathbf{G}_{0, p r i}^{V V}\left(\frac{d_{0}}{d}\right)^{n_{p r i}^{V V}}}_{\mathbf{P}_{p r i}^{V V}(d)}+ \\
& \underbrace{\chi_{s e c}^{V V}(d) \mathbf{G}_{0, s e c}^{V V}\left(\frac{d_{0}}{d}\right)^{n_{s e c}^{V V}}}_{\mathbf{P}_{\text {sec } V}^{V}(d)}+ \\
& \underbrace{\chi_{D M C}^{V V}(d) \mathbf{G}_{0, D M C}^{V V} \mathbf{T}^{V V} e^{\frac{-d}{c \mathbf{T}^{V V}}}}_{\mathbf{P}_{D M C}^{V V}(d)} .
\end{aligned}
$$

The path gains for the DMC with the power law are not detailed here for spacing reasons but could easily be derived. In total, 24 parameters, some of which are distance-dependent, are required to tune the PDP or path gain models whether the DMC follows an exponential or power law model in the delay domain. All parameters are summarized in Table I. Despite the high level of complexity, this approach provides deep information about each mechanism for any polarization link. For instance, the model grants flexibility to derive other desired characteristics such as path loss models for each propagation

\begin{tabular}{|c|c|c|c|c|c|}
\hline \multirow{6}{*}{$n^{X Y}$} & \multirow[b]{2}{*}{ Polar. Link } & \multirow[b]{2}{*}{ Pri. SMC } & \multirow[b]{2}{*}{ Sec. SMC } & \multicolumn{2}{|c|}{ DMC } \\
\hline & & & & $\begin{array}{c}\text { D } \\
\text { Exp. Law }\end{array}$ & Power Law \\
\hline & $H H$ & $n_{p r i}^{\mathrm{HH}}$ & $n_{\mathrm{sec}}^{\mathrm{HH}}$ & $\mathrm{X}$ & $n_{D M C}^{H H}$ \\
\hline & $H V$ & $n_{p r i}^{H V}$ & $n_{\text {sec }}^{H V}$ & $\mathrm{X}$ & $n_{D M C}^{H V}$ \\
\hline & $V H$ & $n_{p r i}^{V H}$ & $n_{\text {sec }}^{V H}$ & $\mathrm{X}$ & $n_{D M C}^{V H}$ \\
\hline & $V V$ & $n_{p r i}^{V V}$ & $n_{\text {sec }}^{V V}$ & $\mathrm{X}$ & $n_{D M C}^{V V}$ \\
\hline \multirow{2}{*}{$\mathbf{G}_{0}^{X Y}$} & $H H$ & $\mathbf{G}_{0, p r i}^{H H}$ & $\mathbf{G}_{0, \mathrm{sec}}^{\mathrm{HH}}$ & $\mathbf{G}_{0, D M C}^{H H}$ & $\mathbf{G}_{0, D M C}^{H H}$ \\
\hline & $V V$ & $\mathbf{G}_{0, p r i}^{V V}$ & $\mathbf{G}_{0, s e c}^{V V}$ & $\mathbf{G}_{0, D M C}^{V V}$ & $\mathbf{G}_{0, D M C}^{V V}$ \\
\hline \multirow{2}{*}{$\chi^{X Y}$} & $H H$ & $\chi_{p r i}^{H H}$ & $\chi_{\text {sec }}^{H H}$ & $\chi_{D M C}^{H H}$ & $\chi_{D M C}^{H H}$ \\
\hline & $V V$ & $\chi_{p r i}^{V V}$ & $\chi_{s e c}^{V V}$ & $\chi_{D M C}^{V V}$ & $\chi_{D M C}^{V V}$ \\
\hline \multirow{4}{*}{$T^{X Y}$} & $H H$ & $\mathrm{X}$ & $\mathrm{X}$ & $T^{H H}$ & $\mathrm{X}$ \\
\hline & $H V$ & $\mathrm{X}$ & $\mathrm{X}$ & $T^{H V}$ & $\mathrm{X}$ \\
\hline & $V H$ & $\mathrm{X}$ & $\mathrm{X}$ & $T^{V H}$ & $\mathrm{X}$ \\
\hline & $V V$ & $\mathrm{X}$ & $\mathrm{X}$ & $T^{V V}$ & $\mathrm{X}$ \\
\hline
\end{tabular}
component which will be later discussed in the paper.

TABLE I

MOdel PARAMETERS

\section{Measurement CAmpaign And Data Processing}

\section{A. Measurement Setup and Scenario}

The validity of the developed model is assessed with data collected by an $8 \times 8 \mathrm{MIMO}$ channel sounder at $1.3 \mathrm{GHz}$ with $22 \mathrm{MHz}$ bandwidth in the atrium $(48.8 \mathrm{~m} \times 36.35 \mathrm{~m} \times 18 \mathrm{~m})$ of the EuraTechnologies Center, Lille (France). Prior being refurbished into a high-technology building, this three levelhigh environment was the host of a textile plant, explaining the presence of traditional brick walls, marble floor, large windows surface, and metallic structure, as depicted in Fig. 2(a). A few chairs, tables, benches, and decoration plants are located around the centre for social events such that the environment can be considered as almost empty. Figure 2(b) presents the 13 LOS Tx-Rx positions in the hall with distances ranging between $10 \mathrm{~m}$ and $45 \mathrm{~m}$. The measurement campaign was performed at night without workers and visitors to ensure that the radio links are static over time. The transmitting unit was moved around the hall whereas the receiving unit was set to the same position for all measurements. A virtual uniform circular array (UCA) was used for both Tx and Rx with dual-polarized patch antennas (antenna Cross-Polar Discrimination XPD > $15 d B$ for the main beam direction) located at $1.6 \mathrm{~m}$ high.

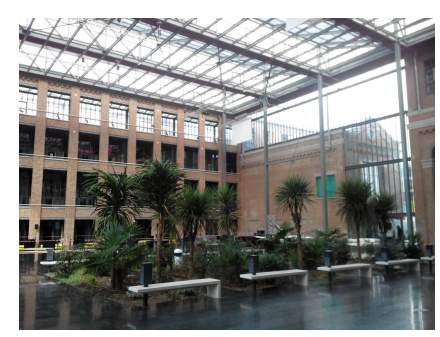

(a)

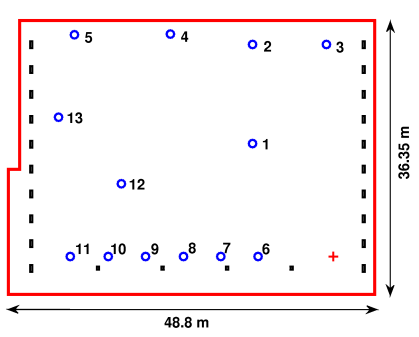

(b)
Fig. 2. (a) Picture of the EuraTechnologies Center atrium (Lille, France) from the receiving array point of view. (b) Tx (blue circles) - Rx (red cross) measurement pairs in the EuraTechnologies Center atrium. The black squares indicate the vertical brick beams visible in (a).

The time-varying complex impulse response of the polarimetric MIMO radio channel $\mathbf{h}^{X Y}(\tau, t, m, n)$ can be obtained by Fourier transforming sampled versions of the frequency response $\mathbf{H}^{X Y}(f, t, m, n)$ measured for all Tx - Rx links:

$$
\mathbf{h}^{X Y}(\tau, t, m, n)=\mathcal{F}^{-1}\left(\mathbf{H}^{X Y}(f, t, m, n)\right),
$$

where $\tau$ is the time-delay, $t$ the sampled time, $f$ the sampled frequency, $m$ the $m^{t h}$ antenna of the Tx array, $n$ the $n^{t h}$ antenna of the $\mathrm{Rx}$ array, and $\mathcal{F}^{-1}$ the inverse Fourier operator. A major benefit of MIMO-based techniques relies in the measurement of a large set of data over frequency, time, and space, depending upon the sounder architecture, antenna array, and investigated scenario. When the channel is not varying with time (i.e., static), the dependence of the radio channel on the transmitter-receiver distance $d$ can also be investigated. The polarimetric distance-dependent PDP $\mathbf{G}^{X Y}(\tau, d)$ can be obtained from the expectation of the squared magnitude of $\mathbf{h}^{X Y}$ :

$$
\mathbf{G}^{X Y}(\tau, d)=\mathbf{E}\left[\left|\mathbf{h}^{X Y}(\tau, d, m, n)\right|^{2}\right] .
$$

The expectation operator is applied over all $\mathrm{Tx}-\mathrm{Rx}$ links to remove the small scale fading. As an example, Fig. 3 presents the measured polarimetric PDP for position 6 (short Tx - Rx distance) and 13 (large $\mathrm{Tx}$ - Rx distance). The exponential decay of the PDP indicates the presence of DMC for all polarization links. In addition, SMC clusters can be clearly 
observed for the co-polar PDP but individual SMC cannot be visually distinguished from each other due to the limited bandwidth.

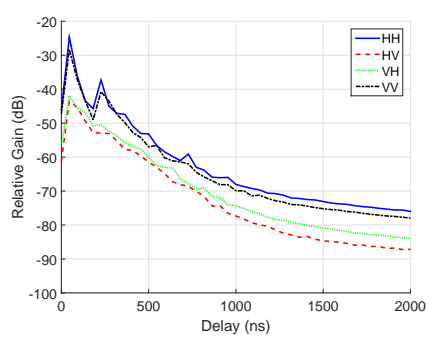

(a)

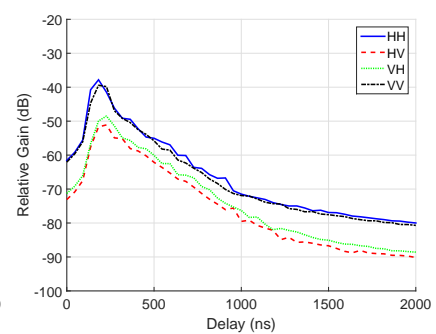

(b)
Fig. 3. Example of measured polarimetric PDP for (a) position 6 and (b) position 13

\section{B. Data Processing}

In order to extract the model parameters from the measured radio channels, it is necessary to fit the path gain model with the SMC and DMC path gain estimates. This is performed with the following procedure:

1) Parametric Estimation: Since we are solely interested into reconstituting estimated versions of the primary/secondary SMC and DMC PDP and subsequent path gain, the polarimetric SMC and DMC parameters have to be initially estimated with a high-resolution parametric estimator. To this end, the data were post-processed with a polarimetric version of the Maximum-Likelihood (ML) parametric estimator RiMAX [12]. For instance, the wideband MIMO measurements performed in this work allow to access the Time of Arrival (TOA), Direction of Arrival (DOA), Direction of Departure (DOD), polarimetric complex gain for each SMC, as well as the DMC. A deeper analysis of the estimates could be performed in terms of geometrical/stochastic channel modeling, clustering, or depolarization mechanisms like in [17] but is outside the scope of this paper. Typically, the sampled array response vector $\mathbf{h}^{X Y}$ can be written as the sum of the SMC $\mathbf{s}^{X Y}$ and DMC $\mathbf{d}^{X Y}$ :

$$
\mathbf{h}^{X Y}=\mathbf{s}^{X Y}+\mathbf{d}^{X Y} .
$$

In addition, the RiMAX data model assumes that the covariance matrix can be factorized into a Kronecker product on the basis that all dimensions are independent [12].

$$
\mathbf{R}=\mathbf{I}_{M_{R}} \otimes \mathbf{I}_{M_{T}} \otimes \mathbf{R}_{f},
$$

where I represents the identity matrix. The dense multipath field is modeled as uncorrelated in the spatial (angular) domains ( $\mathbf{I}_{M_{R}}$ and $\mathbf{I}_{M_{T}}$ ) but correlated in the frequency (timedelay) domain $\left(\mathbf{R}_{f}\right)$. The isotropy assumption in the angular domain implies that the DMC should be antenna independent as demonstrated in [14]. Originally, the DMC is estimated from the covariance matrix of the complete data model (i.e. sum of all polarization links) such that the estimate is an average across all polarization states. However, the DMC power characteristics are typically not uniform across all polarization links like for the investigated scenarios (see Fig. 3).
Consequently, the covariance matrix can be broken down into polarimetric parts such that the DMC can be estimated for each polarization link $X Y$ like reported here [17].

$$
\mathbf{R}^{X Y}=\mathbf{I}_{M_{R}} \otimes \mathbf{I}_{M_{T}} \otimes \mathbf{R}_{f}^{X Y}
$$

In addition, the antennas were de-embedded from the measured radio channels with the sampled polarimetric radiating patterns of the patch antennas. The Effective Aperture Distribution Function (EADF) technique was used to store and interpolate the polarimetric gain for each SMC as a function of azimuth and elevation angles for both DOA and DOD [18]. RiMAX is an iterative algorithm wherein a fixed number of SMC are estimated for each iteration step along with the DMC. Here, the number of new SMC per iteration was set to 5 as originally suggested in [12]. This number was chosen as a good trade-off between the estimator computational time and its ability to split coupled SMC. Note that no significant change in the results was obtained with a larger number. The reliability of each new SMC is checked with an SNR criterion [12], [14] and the algorithm stops itself when all 5 SMC fail the criterion. 50 iterations were chosen such that the maximum number of SMC was potentially 250 . However, the algorithm stopped before reaching this limit for all positions and the mean number of estimated SMC per position was found to be around 75. For the sake of comparison, this value is in-between the number of SMC obtained by ray-tracing in an empty parallelepipedic scenario with a $5^{\text {th }}(61)$ and $6^{\text {th }}$ (85) order of reflection.

2) PDP Estimation: From here, the band-limited $\mathbf{G}_{\text {pri }}^{X Y}$, $\mathbf{G}_{s e c}^{X Y}$, and $\mathbf{G}_{D M C}^{X Y}$ can be reconstructed from the SMC and DMC parameters thanks to the estimator data model. At this point, it is necessary to define which SMC contribute to the primary and secondary components. For indoor scenarios with low room volumes, the LOS and first-order components typically arrive within a short delay period and contribute all to the primary part of the PDP. However, for large volume scenarios such as the one considered in this work, some of the first-order components may arrive much later than the LOS. This is particularly true when the Tx - Rx distance is short like for position 6 (Fig. 3(a)). For this position, peaks are observed $180 \mathrm{~ns}(54 \mathrm{~m})$ later than the LOS and attributed to reflections off the left and up walls. Here, only the SMC which contribute to the first observed peak of the PDP were considered for the primary SMC. This is done by picking all SMC within one delay bin (45 ns) with respect to the LOS and all the remaining SMC are thrown into the secondary SMC. This criterion was found to be simple to implement and in-line with the proposed model. Figure 4 presents the estimated contribution of the primary SMC, secondary SMC, and DMC to the total path gain as a function of distance for all polarimetric links. It is observed that the primary SMC and DMC contribution is not only dependent to the distance but also polarization links as it will be discussed later in Section V-A. In contrast, the contribution of the secondary SMC to the total path gain is weaker than the primary SMC and DMC with less than $9 \%$ on average for the co-polar and $4 \%$ for the cross-polar links, respectively. 


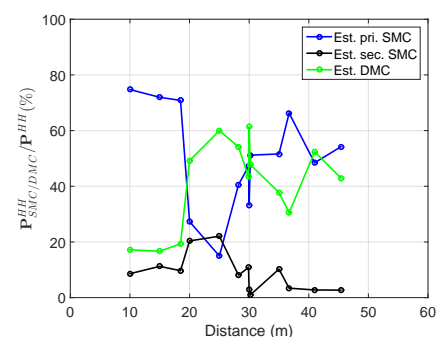

(a)

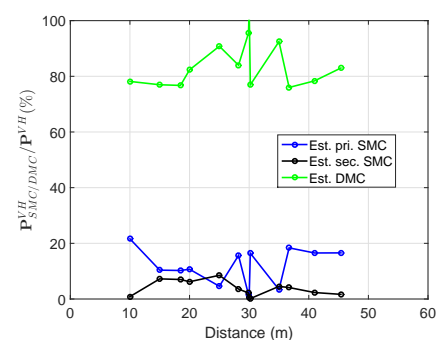

(c)

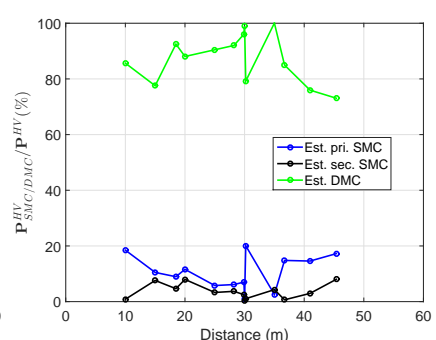

(b)

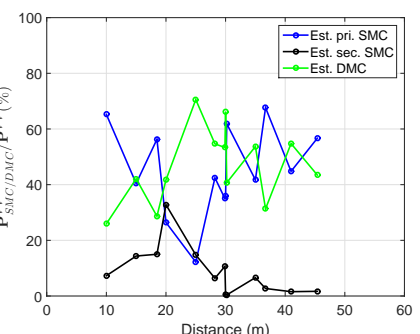

(d)
Fig. 4. Estimated contribution of the primary SMC, secondary SMC, and DMC (in \%) to the total path gain as a function of distance for polarimetric links (a) HH, (b) HV, (c) VH, and (d) VV.

3) SMC and DMC with Power Law: After computing $\mathbf{P}_{\text {pri }}^{X Y}, \mathbf{P}_{\text {sec }}^{X Y}$, and $\mathbf{P}_{D M C}^{X Y}$ from their respective PDP, a linear regression is performed to estimate the polarimetric path loss exponent $n_{p r i}^{X Y}, n_{s e c}^{X Y}$, and $n_{D M C}^{X Y}$, respectively. $\chi_{S M C}^{H H}, \chi_{S M C}^{V V}$, $\chi_{D M C}^{H H}$, and $\chi_{D M C}^{V V}$, are obtained indirectly as a function of the Cross-Polar Discrimination (XPD) and it can be shown that:

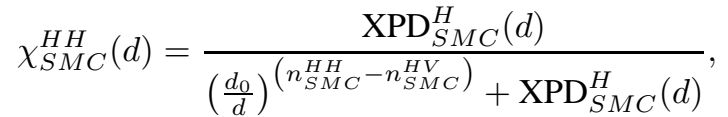

$$
\begin{aligned}
& \chi_{S M C}^{V V}(d)=\frac{\operatorname{XPD}_{S M C}^{V}(d)}{\left(\frac{d_{0}}{d}\right)^{\left(n_{S M C}^{V V}-n_{S M C}^{V H}\right)}+\operatorname{XPD}_{S M C}^{V}(d)},
\end{aligned}
$$

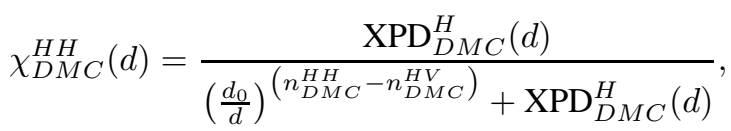

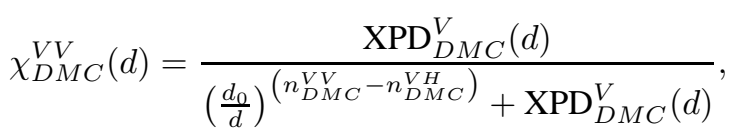

where the distance-dependent XPD with respect to $H$ and $V$ is computed from the estimated path gain for the SMC (pri or $s e c$ ) or DMC by:

$$
\begin{aligned}
& \operatorname{XPD}_{S M C / D M C}^{H}(d)=\left(\frac{\mathbf{P}_{S M C / D M C}^{H H}(d)}{\mathbf{P}_{S M C / D M C}^{H V}(d)}\right), \\
& \operatorname{XPD}_{S M C / D M C}^{V}(d)=\left(\frac{\mathbf{P}_{S M C / D M C}^{V V}(d)}{\mathbf{P}_{S M C / D M C}^{V H}(d)}\right) .
\end{aligned}
$$

Finally, the reference gain for the primary/secondary SMC and DMC are computed at reference distance $d_{0}$ from:

$$
\mathbf{G}_{0, S M C / D M C}^{H H}=\frac{\mathbf{P}_{S M C / D M C}^{H H}\left(d_{0}\right)}{\chi_{S M C / D M C}^{H H}\left(d_{0}\right)},
$$

$$
\mathbf{G}_{0, S M C / D M C}^{V V}=\frac{\mathbf{P}_{S M C / D M C}^{V V}\left(d_{0}\right)}{\chi_{S M C / D M C}^{V V}\left(d_{0}\right)} .
$$

4) DMC with Exponential Model: Similarly, provided that $\mathbf{T}^{X Y}$ is a by-product of the estimator, $\chi_{D M C}^{H H}$ and $\chi_{D M C}^{V V}$ are given by:

$$
\begin{aligned}
& \chi_{D M C}^{H H}(d)=\frac{\mathrm{XPD}_{D M C}^{H}}{\left(\frac{\mathbf{T}^{H H}}{\mathbf{T}^{H V}}\right) e^{\frac{-d}{c}\left(\frac{\mathbf{T}^{H V}-\mathbf{T}^{H H}}{\mathbf{T}^{H V} \mathbf{T}^{H H}}\right)}+\mathbf{X P D}_{D M C}^{H}}, \\
& \chi_{D M C}^{V V}(d)=\frac{\mathbf{X P D}_{D M C}^{V}}{\left(\frac{\mathbf{T}^{V V}}{\mathbf{T}^{V H}}\right) e^{\frac{-d}{c}\left(\frac{\mathbf{T}^{V H}-\mathbf{T}^{V V}}{\mathbf{T}^{V V} \mathbf{T}^{V H}}\right)}+\mathbf{X P D}_{D M C}^{V}},
\end{aligned}
$$

where $\mathrm{XPD}_{D M C}^{H}$ and $\mathrm{XPD}_{D M C}^{H}$ are also computed with Eq. (37)-(38). Finally, $\mathbf{G}_{0, D M C}^{H H}$ and $\mathbf{G}_{0, D M C}^{V V}$ are computed at reference distance $d_{0}$ from:

$$
\begin{aligned}
\mathbf{G}_{0, D M C}^{H H} & =\frac{\mathbf{P}_{D M C}^{H H}\left(d_{0}\right)}{\chi_{D M C}^{H H}\left(d_{0}\right) \mathbf{T}^{H H} e^{\frac{-d_{0}}{c \mathbf{T}^{H H}}}}, \\
\mathbf{G}_{0, D M C}^{V V} & =\frac{\mathbf{P}_{D M C}^{V V}\left(d_{0}\right)}{\chi_{D M C}^{V V}\left(d_{0}\right) \mathbf{T}^{V V} e^{\frac{-d_{0}}{c \mathbf{T}^{V V}}}} .
\end{aligned}
$$

\section{RESUlTS AND DisCUSSION}

\section{A. Model Parameters}

First of all, the polarimetric path loss exponents were fitted from the estimated primary, secondary SMC, and DMC path gains. Then, the polarization coefficients as well as the reference gains were computed for the SMC and DMC from (33)-(44). It is shown in Fig. 5(a) and (b) that the primary and secondary SMC do not exhibit strong depolarization effects (i.e. $\chi_{S M C}^{X Y} \approx 1$ ) over the whole distance range. In contrast, a larger depolarization is observed for the DMC but still constant. It is only noted a slight change trend for the DMC with exponential model, greater for VV than for $\mathrm{HH}$. This change is too weak to be considered and the complexity of the model can be decreased by taking the averaged value over the studied distance range. Similarly, $\mathbf{T}^{X Y}$ was not found to be distance-dependent across all polarization links. A standard deviation of $14.7 \mathrm{~ns}, 3.7 \mathrm{~ns}, 2.7 \mathrm{~ns}$, and $5.5 \mathrm{~ns}$ was computed for $H H, H V, V H$, and $V V$, respectively. Hence, the averaged value was also selected to tune the model. Moreover, this confirms the validity of the room electromagnetics theory and applicability of the proposed model but also that the estimation of $\mathbf{T}$ is more faithful for the cross-polar links. Finally, the reference gains were computed from the data at reference distance $d_{0}=10 \mathrm{~m}$ for the SMC and DMC with the power law and $d_{0}=41 \mathrm{~m}$ for the DMC with the exponential law, respectively. Table II summarizes the complete set of parameters required to tune the proposed PDP and path gain model for the investigated scenario.

\section{B. Model Validation}

Figure 6 presents a comparison between the estimated and modeled path gain for each propagation mechanism and polarization link. In addition, Fig. 7 presents the distancedependent total path gain of the estimated and modeled data, 


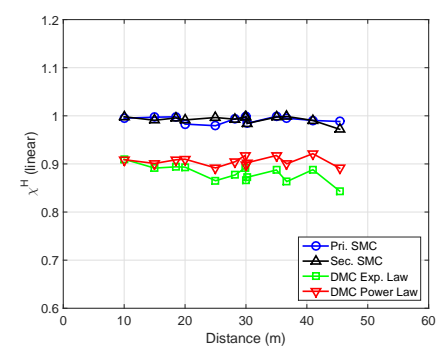

(a)

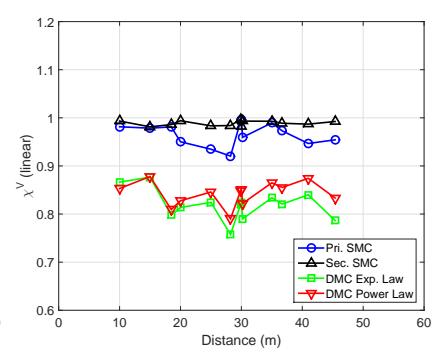

(b)
Fig. 5. Primary/secondary SMC and DMC polarization coefficients for (a) $H$ and (b) $V$.

TABLE II

Estimated MODEL PARAMETERS

\begin{tabular}{|c|c|c|c|c|c|c|c|c|c|c|c|c|}
\hline & \multirow{2}{*}{\multicolumn{3}{|c|}{ Pri. SMC }} & \multirow{2}{*}{\multicolumn{3}{|c|}{ Sec. SMC }} & \multicolumn{6}{|c|}{ DMC } \\
\hline & & & & & & & & Power Law & & & Exp. Law & \\
\hline & $n_{p r i}^{X Y}$ & $\mathrm{G}_{0, p r i}^{X Y}(\mathrm{~dB})$ & $\chi_{p r i}^{X Y}$ & $\begin{array}{l}n_{s e c}^{X Y} \\
x y\end{array}$ & $\mathbf{G}_{0, s e c}^{X Y}(\mathrm{~dB})$ & $\chi_{X_{s e c}^{X Y}}^{X Y}$ & \begin{tabular}{|l|l|}
$n_{D M C}^{X Y}$ \\
\end{tabular} & $\mathbf{G}_{0, D M C}^{X Y}(\mathrm{~dB})$ & $\chi_{D M C}^{X Y}$ & $T^{T^{X Y}(n s)}$ & $\mathbf{G}_{0, D M C}^{X Y}(\mathrm{~dB})$ & $x_{D M C}^{X Y}$ \\
\hline$H H$ & 2.19 & -0.9 & \begin{tabular}{|l|}
0.99 \\
\end{tabular} & 2.88 & -10.3 & 0.99 & 1.14 & -6.88 & 0.91 & 125.6 & 60.9 & 0.88 \\
\hline$H V$ & 1.26 & $\mathrm{x}$ & $\mathrm{x}$ & 1.26 & $\mathrm{x}$ & $x$ & 0.85 & $\mathrm{x}$ & $\mathrm{x}$ & 126.6 & $\mathrm{x}$ & $\mathrm{x}$ \\
\hline$V H$ & 1.11 & $x$ & $x$ & 1.11 & $x$ & $\frac{x}{x}$ & 0.78 & $\mathrm{x}$ & $\mathrm{x}$ & 126.6 & $\mathrm{x}$ & $x$ \\
\hline$V V$ & 1.56 & -4.6 & \begin{tabular}{|l|l|}
0.97 \\
\end{tabular} & 3.16 & -14.2 & 0.99 & 1.11 & -8 & 0.84 & 116 & 60.2 & 0.82 \\
\hline
\end{tabular}

respectively. The path gain models were built from (24)-(27) with the parameters listed in Table II. The results show a good agreement between the model and data estimated from the measurements. The goodness-of-fit is demonstrated by the root-mean-square error (RMSE) values computed between the path gain deduced from the models and measurements (in dB) for each polarization link and shown in Table III. The RMSE values are found as good with a power law or an exponential model for the investigated range. It is observed larger RMSE values for the cross-polar primary SMC than for the co-polar links. Conversely, smaller RMSE values are obtained for the cross-polar DMC than for the cross-polar links. The secondary SMC present almost the largest RMSE values across the polarization links. The large RMSE values for the SMC could be attributed to the time-gating approach used to select the SMC which has not been optimized. It is noteworthy a deeper analysis of the polarization mechanisms for each component is not discussed here since the paper primarly focuses on developing and validating the model.

TABLE III RMSE $[d B]$ OF PATH GAINS

\begin{tabular}{|c|c|c|c|c|c|c|}
\cline { 3 - 7 } \multicolumn{2}{c|}{} & \multicolumn{2}{c|}{$\mathbf{P}_{D M C}^{X Y}$} & \multicolumn{2}{c|}{$\mathbf{P}^{X Y}$} \\
\cline { 2 - 7 } & $\mathbf{P}_{\text {pri }}^{X Y}$ & $\mathbf{P}_{\text {sec }}^{X Y}$ & Exp. Model & Power Law & Exp. Model & Power Law \\
\hline \hline$H H$ & 3.41 & 4.31 & 1.16 & 0.72 & 1.76 & 1.75 \\
\hline$H V$ & 4.64 & 4.32 & 0.74 & 0.63 & 0.81 & 0.7 \\
\hline$V H$ & 5.65 & 6.45 & 0.73 & 0.63 & 0.46 & 0.48 \\
\hline$V V$ & 3.55 & 6.73 & 1.53 & 1.22 & 1.39 & 1.42 \\
\hline
\end{tabular}

\section{SMC AND DMC PATH LOSS MODELS}

The development of faithful path loss models is critical to the deployment of wireless systems in indoor scenarios. For instance, indoor propagation models rely on the empirical path loss exponent $n$ which links the received power with

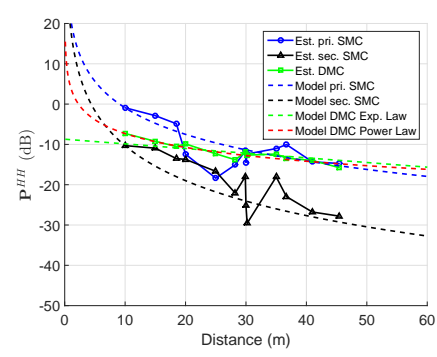

(a)

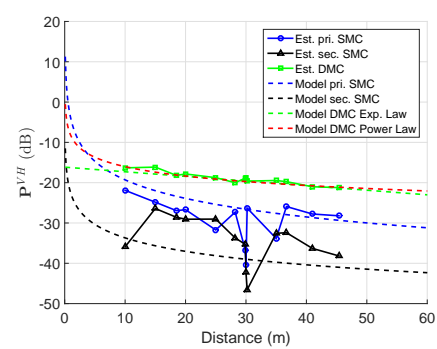

(c)

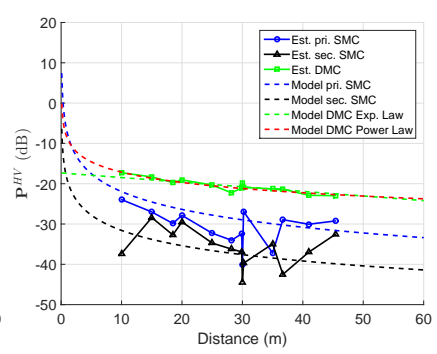

(b)

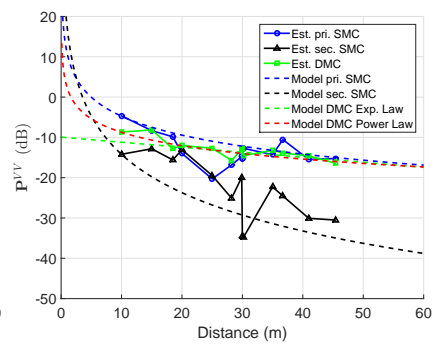

(d)
Fig. 6. Estimated and modeled primary/secondary and DMC path gain as a function of distance for polarimetric links (a) $\mathrm{HH}$, (b) $\mathrm{HV}$, (c) $\mathrm{VH}$, and (d) VV.

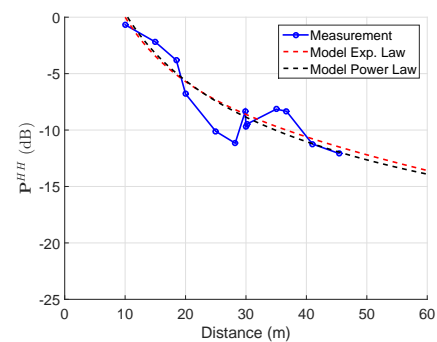

(a)

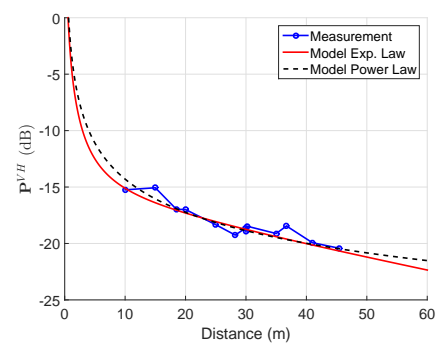

(c) (b)

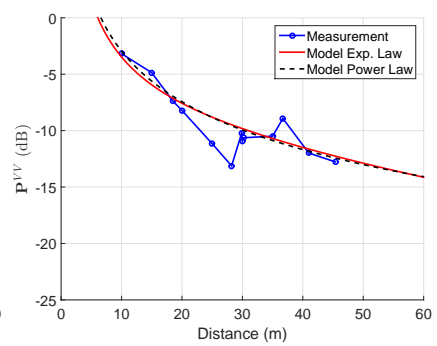

(d)

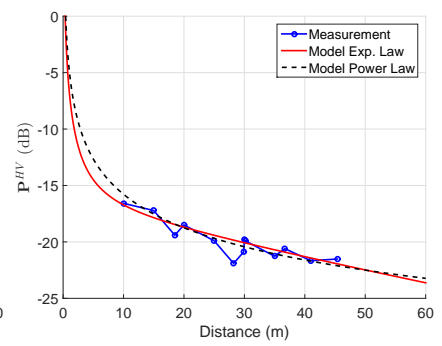

Fig. 7. Estimated and modeled total path gain as a function of distance for polarimetric links (a) $\mathrm{HH}$, (b) $\mathrm{HV}$, (c) $\mathrm{VH}$, and (d) VV.

the logarithm of the distance [1]. A single slope model is typically observed but multi-slope (or multiple breakpoint) path loss models were also reported in office scenarios when the distance was large or when the receiver (transmitter) was located at a different floor [19]. Obviously, the path loss exponent retrieved from the measured channels must be a path loss weighted by the contribution of the primary/secondary SMC and DMC if present; contribution which will depend on the Tx - Rx distance, room configuration, shadowing condition, etc. We note that this aspect is not well addressed 
in the literature. Moreover, DMC path loss models are missing to the knowledge of the authors. Hence, the computation of the DMC to the total power ratio is a prerequisite to analyse the contribution of each mechanism to the radio channel and impact on the path loss exponent.

\section{A. Polarimetric Reverberation Ratio}

Similarly to previous works [14], [17], [20], a polarimetric DMC to total power ratio (or reverberation ratio) $\mathbf{R}^{X Y}(d)$ can be derived:

$$
\mathbf{R}^{X Y}(d)=\frac{\mathbf{P}_{D M C}^{X Y}(d)}{\mathbf{P}^{X Y}(d)} .
$$

Figure 8 presents the polarimetric reverberation ratio $\mathbf{R}^{X Y}$ as a function of distance computed with the data and model in which the power law and exponential model were both considered. A good agreement is found between the measured and modeled data for the investigated distance range. Results with the exponential and power laws are similar for distances up to $50 \mathrm{~m}$ but differ greatly for larger distances. As originally shown in [16] with an exponential model, $\mathbf{R}^{X Y}$ tends towards zero when the distance between the transmitter and receiver exceeds several hundred meters. However, the behavior of $\mathbf{R}^{X Y}$ is rather different with the power law and converges towards unity without passing through a maximum value. For the exponential model, the distance at which $\mathbf{R}^{X Y}$ is maximal equals $50 \mathrm{~m}$ for both $H V$ and $V H$ but equals $75 \mathrm{~m}$ for $H H$ and $50 \mathrm{~m}$ for $V V$. However, it is noted that it is not possible at this point to experimentally verify which model holds for longer distances.

In contrast to the path gain models, a strong asymmetry (shape and distance at which the maximum value is obtained) is observed between all polarimetric subchannels and, in particular, the co-polar ones. The fact that reverberation effects are non-uniform across all polarization links highlights the complexity of propagation mechanisms even in simple scenarios and that polarimetric radio channel models including DMC must be designed with care. The measured reverberation ratio values are ranging between $20 \%$ and $75 \%$ for $\mathbf{R}^{H H}$ and $\mathbf{R}^{V V}$ similarly to other investigated indoor scenarios ([2], [13], [14], [17] and above $75 \%$ up to $100 \%$ for $\mathbf{R}^{H V}$ and $\mathbf{R}^{V H}$. High reverberation ratio values are expected in indoor or industrial scenarios (when the room electromagnetics theory applies) since intermediate distances are typically used. Consequently, this result confirms the findings of all previous studies on this topic.

\section{B. Path Loss Models with DMC}

1) DMC only: First, it is considered that the radio channel only includes DMC or that the contribution of the SMC is weak compared to DMC. This case could happen if shadowing conditions are harsh (strong Non-Line-Of-Sight NLOS) or if polarization coefficients are close to unity like in this work (Fig. 8(b) and (c)). For the latter case, the path gain model for the channel could be simplified by taking into account only the DMC path gain. For instance, the polarimetric path loss $\mathbf{P L} \mathbf{L}_{D M C}^{X Y}$ are obtained from the DMC path gain models

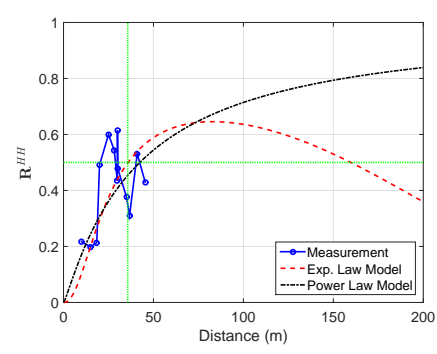

(a)

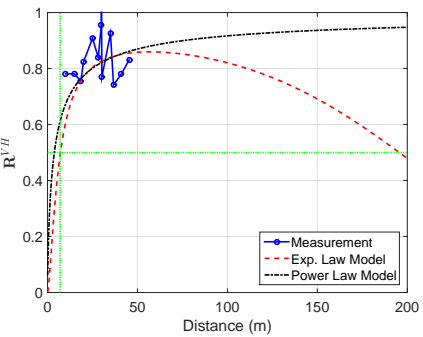

(c)

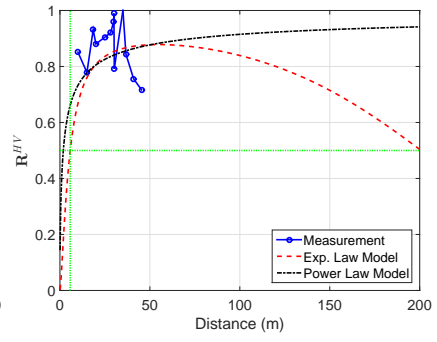

(b)

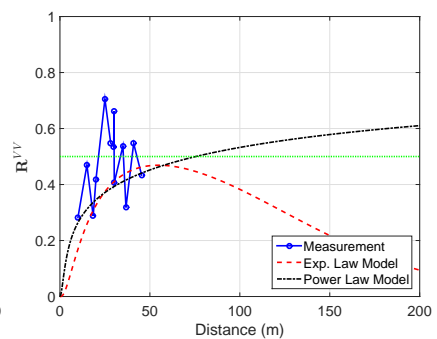

(d)
Fig. 8. $\mathbf{R}^{X Y}$ as a function of distance for (a) $H H$, (b) $H V$, (c) $V H$, and (d) $V V$.

$\mathbf{P}_{D M C}^{X Y}$. Only the cross-polar links $H V$ with exponential law are treated in the discussion but the other links and model can be derived in the similar fashion:

$$
\left[\mathbf{P L}_{D M C}^{H V}(d)\right]_{d B}=-\left[\left(1-\chi_{D M C}^{H H}\right) \mathbf{G}_{0, D M C}^{H H} \mathbf{T}^{H V}\right]_{d B}+\frac{10 d}{c \mathbf{T}^{H V} \log (10)} .
$$

Provided that $\mathbf{T}^{H V}$ and $\chi_{D M C}^{H V}$ are constant like in this work, it follows that the DMC path loss is linear with the distance and inversely proportional to $\mathbf{T}^{H V}$. This proportionality parameter or DMC path loss factor denoted here $\eta_{D M C}^{H V}$ is given by:

$$
\eta_{D M C}^{H V}=\frac{10}{c \mathbf{T}^{H V} \log (10)} .
$$

Equation (47) establishes a direct relationship between $\mathbf{T}^{H V}$ and $\eta_{D M C}^{H V}$ and provides an attractive approach to link the room electromagnetics theory characterized by $\mathbf{T}^{H V}$ with the loss mechanisms of the channel characterized by the DMC path loss factor. Evidently, $\eta_{D M C}^{X Y}$ will also change if the room electromagnetics theory does not apply $\left(\mathbf{T}^{X Y}\right.$ not constant across all polarization links). Finally, it is possible to reformulate the DMC path gain model by substituting (47) in (19) to highlight $\eta_{D M C}^{H V}$ :

$$
\mathbf{P}_{D M C}^{H V}(d)=\left(\left(1-\chi_{D M C}^{H H}\right) \mathbf{G}_{0, D M C}^{H H} \mathbf{T}^{H V}\right) e^{\frac{-\eta_{D M C}^{H V} d . l_{0 g_{10}}}{10}} .
$$

2) $S M C$ and $D M C$ : The more general case appears when the primary SMC and DMC are both included into the radio channel like the co-polar links in this work (Fig. 8(a) and (d)). As an example (all other polarization can be similarly derived), the path loss model for $H H$ is given by:

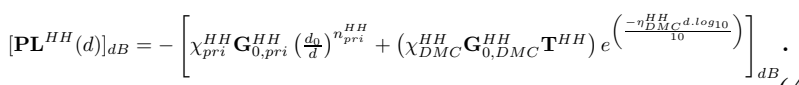

The secondary SMC was omitted in Eq. (49) since its contribution to the radio channel is marginal. This expression provides more insight about the path loss mechanisms of 
the radio channel and highlights the competition between the two components. For instance, when the transmitter-receiver distance is really short or really large (Fig. 8), the primary SMC dominates the DMC and the contribution of the DMC to the path loss is small. Conversely, there is a transmitterreceiver distance range for which the DMC is the dominant propagation mechanism such that the contribution of the primary SMC is now small. If the DMC is modeled with a power law, then the primary SMC dominates the DMC only when the transmitter-receiver distance is short.

\section{Path Loss Parameters Fitting Technique}

The presented path loss models require the a-priori estimation of the SMC and DMC path gains from the measured polarimetric radio channels. It is reminded that the estimation process is a highly complex mathematical treatment requiring huge computational post-processing time especially when the dataset comprises hundreds or even thousands measurement points. In addition, modeling errors due to the antenna array calibration or assumptions in the data model could result in degraded estimates [18]. In contrast, classical log-distance path loss models have been widely used because they follow the opposite philosophy. They don't make any assumptions at all about the propagation mechanisms and the fitting steps exhibit low complexity and fast processing time characteristics. Here, we explore the possibility to use the SMC plus DMC path loss models as fitting metrics for the measurement data. The motivation is to develop a low complexity technique to obtain joint estimates of the SMC and DMC path loss parameters without the need for estimation technique.

The joint analysis of the reverberation results and SMC plus DMC path loss models reveals that the characterization of the path loss parameter for each propagation mechanism can be split into two fitting steps. Two cases can be distinguished in Fig. 8. In a first case, $\mathbf{R}^{X Y}$ is below 0.5 (see horizontal line) such that the SMC power is larger than that of the DMC (copolar links). For the second case, $\mathbf{R}^{X Y}$ is above 0.5 such that the DMC power is now much larger than that of the SMC (cross-polar links).

As an example, Fig. 9 illustrates this two-step fitting procedure for the measured $H H$ radio channel data. In a first step (Fig. 9(a)), the path loss exponent for the primary SMC noted $n_{p r i}^{H H-I}$ is computed from the linear region of the log-distance curve for distances where the SMC gain is greater than the DMC. In a second step (Fig. 9(b)), the DMC path loss factor $\eta_{D M C}^{H H-I I}$ is computed from the linear region of the lineardistance curve where the DMC gain is greater than the SMC. It is observed that the fitting slope follows well the asymptotic behavior of the model for each region (power law for the SMC and exponential law for the DMC). In addition, the SMC to DMC transition distance between the two propagation mechanisms can be obtained with the intersection of the logand linear-distance lines. For this case, a transition distance $d=37 \mathrm{~m}$ is obtained in agreement with the value in Fig. 8(a) (see vertical line).

In summary, Table IV presents the SMC path loss exponent $n_{p r i}^{X Y-I}$ and DMC path loss factor $\eta_{D M C}^{X Y-I I}$ fitted from this

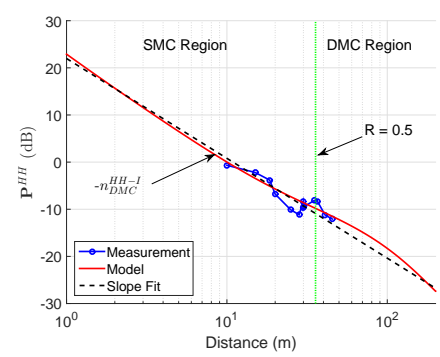

(a)

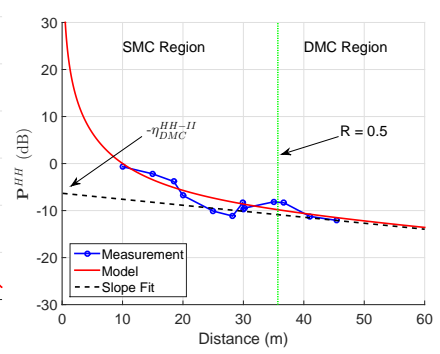

(b)
Fig. 9. Example of the two-step path loss exponent fitting for the $H H$ radio channel. The dotted line (black) is the slope fit for (a) the SMC $\left(n_{p r i}^{\mathrm{HH}-I}\right)$ and (b) DMC $\left(\eta_{D M C}^{H H-I I}\right)$. The vertical line indicates the transition distance at which $\mathbf{R}=0.5$ (37 $\mathrm{m}$ here).

procedure for each polarization link. For the sake of comparison, $n^{X Y}, n_{p r i}^{X Y}, n_{D M C}^{X Y}$ (Table II), and $\eta_{D M C}^{X Y}$ computed with Eq. (47) from the values of $\mathbf{T}_{D M C}^{X Y}$ (Table II) were also added. As discussed at the beginning of this section, it is confirmed that $n^{X Y}$ is in-between $n_{p r i}^{X Y}$ and $n_{D M C}^{X Y}$ but also in line with values reported in [1], [17], [21]. Hence, the need for path loss models where DMC is included is clearly demonstrated. Furthermore, a relatively good agreement is obtained between $n_{p r i}^{X Y}$ with $n_{p r i}^{X Y-I}$ and an excellent fit is obtained between $n_{D M C}^{X Y}$ and $\eta_{D M C}^{X Y-I I}$. This latter result indicates that the reverberation time can be estimated with great accuracy for the cross-polar channels from Eq. (47) without applying complex parametric estimation techniques.

Overall, the results show that it is possible to grasp the path loss characteristics of each mechanism with the twostep fitting approach which is a strong contribution of this work. Finally, even if the investigated distance span and related measurement dataset were limited, it was sufficient to develop the model since the measured data overlap over both regions. This method is general and could be applied to any path loss data already collected in indoor environments wherein room electromagnetics applies.

TABLE IV

PATH LOSS PARAMETERS

\begin{tabular}{c|c|c|c|c|c|c|}
\cline { 2 - 7 } \multicolumn{1}{c|}{} & Meas. & \multicolumn{2}{|c|}{ Pri. SMC } & \multicolumn{3}{c|}{ DMC } \\
\cline { 2 - 7 } & $n^{X Y}$ & $n_{p r i}^{X Y}$ & $n_{p r i}^{X Y-I}$ & $n_{D M C}^{X Y}$ & $\eta_{D M C}^{X Y}$ & $\eta_{D M C}^{X Y-I I}$ \\
\hline \hline$H H$ & 1.63 & 2.19 & 2.17 & 1.14 & 0.12 & 0.17 \\
\hline$H V$ & 0.88 & 1.47 & 0.95 & 0.85 & 0.11 & 0.11 \\
\hline$V H$ & 0.86 & 1.53 & 0.9 & 0.78 & 0.11 & 0.11 \\
\hline$V V$ & 1.21 & 1.56 & 1.89 & 1.11 & 0.13 & 0.17 \\
\hline
\end{tabular}

\section{CONCLUSIONS}

A comprehensive polarimetric distance-dependent model of the PDP and path gain is proposed for large hall scenarios including DMC. The model is validated with polarimetric measurements of a large hall radio channel under LOS conditions at $1.3 \mathrm{GHz}$. The measured MIMO channels were processed by RiMAX to separate the polarimetric primary/secondary SMC 
and DMC from which the parameters of the path gain model were retrieved. The validity and robustness of the proposed approach is provided by the good agreement between the polarimetric data and models. In particular, the description of the radio channel with path loss models is discussed for cases when the DMC is included. Furthermore, a two-step method to compute the joint path loss characteristics of the SMC and DMC directly from the measured data has been developed and validated. In conclusion, the article highlights the need to include a complete polarimetric description of both the SMC and DMC into polarimetric radio channel models such as the COST2100 [22] but also that polarimetric SMC plus DMC path loss models must be carefully thought. Future work will focus on checking the validity of the exponential model or power law for larger Tx - Rx distances.

\section{ACKNOWLEDGMENT}

E. Tanghe is a Post-Doctoral Fellow of the FWO-V (Research Foundation - Flanders). This work was financially supported by the INTERREG IVa project 1.1.7 WiSE and the project IAP BESTCOM, BElgian network on STochastic modelling, analysis, design and optimization of COMmunication systems. This research was also partly funded by the Fund for Scientific Research - Flanders (FWO-V, Belgium) project G.0325.11N and the FORWARD project co-funded by iMinds, a digital research institute founded by the Flemish Government. Project partners are ArcelorMittal, Egemin, Excentis, Siemens, and Volvo, with project support from IWT.

\section{REFERENCES}

[1] T. Rappaport, Wireless Communications: Principles and Practice, 2nd ed. Upper Saddle River, NJ, USA: Prentice Hall PTR, 2001.

[2] J. Poutanen, J. Salmi, K. Haneda, V. Kolmonen, and P. Vainikainen, "Angular and shadowing characteristics of dense multipath components in indoor radio channels," IEEE Trans. Antennas Propag., vol. 59, no. 1, pp. 245-256, 2011.

[3] F. Mani, F. Quitin, and C. Oestges, "Directional spreads of dense multipath components in indoor environments: Experimental validation of a ray-tracing approach," IEEE Trans. Antennas Propag., vol. 60, no. 7, pp. 3389-3396, 2012.

[4] H. Liu, H. Darabi, P. Banerjee, and J. Liu, "Survey of wireless indoor positioning techniques and systems," Systems, Man, and Cybernetics, Part C: Applications and Reviews, IEEE Transactions on, vol. 37, no. 6, pp. 1067-1080, Nov 2007.

[5] A. Bamba, W. Joseph, G. Vermeeren, E. Tanghe, D. P. Gaillot, J. B. Andersen, J. d. Nielsen, M. Lienard, and L. Martens, "Validation of experimental whole-body SAR assessment method in a complex indoor environment," Bioelectromagnetics, vol. 34, no. 2, pp. 122-132, February 2013.

[6] C. Holloway, M. Cotton, and P. McKenna, "A model for predicting the power delay profile characteristics inside a room," IEEE Trans. Veh. Commun., vol. 48, no. 4, pp. 1110-1120, Jul 1999.

[7] R. Rudd, "The prediction of indoor radio channel impulse response," in Antennas and Propagation, 2007. EuCAP 2007. The Second European Conference on, Nov 2007, pp. 1-4.

[8] J. Andersen, J. Nielsen, G. Pedersen, G. Bauch, and M. Herdin, "Room electromagnetics," Antennas and Propagation Magazine, IEEE, vol. 49, no. 2, pp. 27-33, April 2007.

[9] J. Nielsen, J. Andersen, G. Pedersen, and M. Pelosi, "On polarization and frequency dependence of diffuse indoor propagation," in Vehicular Technology Conference (VTC Fall), 2011 IEEE, Sept 2011, pp. 1-5.

[10] D. A. Hill, "Electromagnetic Fields in Cavities. Deterministic and Statistical Theories", W.-I. Press, Ed. John Wiley \& Sons, Inc., 2009.

[11] O. Delangre, "Radio channel characterization and models in reverberating rooms," Ph.D. dissertation, University of Lille1, Villeneuve d'Ascq, FR and Universit Libre de Bruxelles, Brussels, BE, 2008.
[12] A. Richter, "Estimation of radio channel parameters : Models and algorithms," Ph.D. dissertation, Technische Universität Ilmenau, Fakültat für Elektrotechnik und Informationstechnik, Ilmenau, DE, 2005.

[13] F. Quitin, C. Oestges, F. Horlin, and P. De Doncker, "Diffuse multipath component characterization for indoor MIMO channels," in Antennas and Propagation (EuCAP), 2010 Proceedings of the Fourth European Conference on, April 2010, pp. 1-5.

[14] E. Tanghe, D. P. Gaillot, M. Lienard, L. Martens, and W. Joseph, "Experimental analysis of dense multipath components in an industrial environment," IEEE Trans. Antennas Propag., vol. 62, no. 7, pp. 37973805, July 2014.

[15] J. Salmi, J. Poutanen, K. Haneda, A. Richter, V.-M. Kolmonen, P. Vainikainen, and A. Molisch, "Incorporating diffuse scattering in geometry-based stochastic MIMO channel models," in Antennas and Propagation (EuCAP), 2010 Proceedings of the Fourth European Conference on, April 2010, pp. 1-5.

[16] G. Steinbock, T. Pedersen, B. Fleury, W. Wang, and R. Raulefs, "Distance dependent model for the delay power spectrum of in-room radio channels," IEEE Trans. Antennas Propag., vol. 61, no. 8, pp. 43274340, Aug 2013.

[17] D. Gaillot, E. Tanghe, W. Joseph, P. Laly, V.-C. Tran, M. Lienard, and L. Martens, "Polarization properties of specular and dense multipath components in a large industrial hall," IEEE Trans. Antennas Propag., vol. 63 , no. 7 , pp. 3219-3228, July 2015.

[18] M. Landmann, "Limitations of experimental channel characterisation," Ph.D. dissertation, Technische Universität Ilmenau, Fakültat für Elektrotechnik und Informationstechnik, Ilmenau, DE, 2008.

[19] D. Akerberg, "Properties of a tdma pico cellular office communication system," in Globecom 1988, IEEE, December 1988, pp. 1343-1349.

[20] F. Quitin, C. Oestges, F. Horlin, and P. De Doncker, "Polarization measurements and modeling in indoor NLOS environments," IEEE Trans. Wireless Commun., vol. 9, no. 1, pp. 21-25, January 2010.

[21] D. Xu, J. Zhang, X. Gao, P. Zhang, and Y. Wu, "Indoor office propagation measurements and path loss models at $5.25 \mathrm{GHz}$," in Vehicular Technology Conference, 2007. VTC-2007 Fall. 2007 IEEE 66th, Sept 2007, pp. 844-848.

[22] L. Liu, C. Oestges, J. Poutanen, K. Haneda, P. Vainikainen, F. Quitin, F. Tufvesson, and P. Doncker, "The COST 2100 MIMO channel model," Wireless Communications, IEEE, vol. 19, no. 6, pp. 92-99, December 2012. 